\title{
Automated detection of lung cancer using statistical and morphological image processing techniques
}

\author{
Amjed S. Al-Fahoum¹, Eslam B. Jaber², Mohammed A. Al-Jarrah² \\ 1. Biomedical Systems and Informatics Engineering Dept., Hijjawi Faculty for Engineering Technology, Yarmouk \\ University, Irbid 21163 - Jordan. 2. Computer Engineering Dept., Hijjawi Faculty for Engineering Technology, Yarmouk \\ University, Irbid 21163 - Jordan.
}

Correspondence: Amjed S. Al-Fahoum. Address: Biomedical systems and Informatics Engineering Department, Hijjawi Faculty for Engineering Technology, Yarmouk University, Irbid 21163, Jordan. E-mail: afahoum@yu.edu.jo

Received: October 28, 2013

Accepted: March 2, 2014

Online Published: March 13, 2014

DOI : $10.5430 / j b g c . v 4 n 2 p 33$

URL: http://dx.doi.org/10.5430/jbgc.v4n2p33

\begin{abstract}
Lung cancer represents the second most commonly diagnosed cancer among Jordanian population. Evidence that early detection of lung cancer may allow for more timely therapeutic intervention has provided the momentum for lung cancer screening programs around the world. In this study, a computer aided detection (CAD) system is proposed in an attempt to detect the lung cancer areas using computed tomography (CT) images. It is implemented as a "second reader" to help radiologists focus their attention on regions that might be missed during visual interpretation. The proposed CAD system has three main stages; Segmentation by thresholding the CT images, labeling the founded regions and then extracting some diagnostic features of each region for further analysis and interpretation. The study is trained, tested, and validated using images obtained from forty five patients. The obtained results perfectly match the radiologist's diagnosis in detecting the defected areas and quantitatively measuring its size, location, borders as well as displaying its other diagnostic characteristics. Moreover, the proposed system can detect misclassified regions.
\end{abstract}

\section{Key words}

Computer tomography, Labeling, Lung cancer, Thresholding

\section{I ntroduction}

Cancer is the second most frequent cause of death after heart diseases, and it is a major cause of morbidity among Jordanian population ${ }^{[1,2]}$.

Lung cancer constitutes around $10.9 \%$ of all diagnosed cancer cases in Jordan. Lung cancer in males forms $80 \%$ of all lung cancer, where it is $20 \%$ in females. In males, lung cancer ranks the first among all cancer types.

Lung cancer is known as one of the most difficult cancers to cure. The detection of lung cancer in its early stage can be helpful for medical treatment to limit the danger. A conventional technique that assists the detection uses computed tomography (CT) scan, which provides information of three-dimensional (3D) cross sectional images of the lung. A large set of CT images requires radiologists to spend a considerable amount of time interpreting the images. As a means to reduce radiologists' workload, computer aided detection (CAD) systems may be used. The CAD systems can also help 
improving a radiologist's performance in the detection of primary lung cancer since the CAD system can be implemented as a "second reader"; it is expected to help radiologists focus their attention on regions that might contain lung cancer.

Various investigators have developed a number of methods for the automated detection of lung nodules in CT scans. Advances in image reconstruction algorithms and the availability of digitized modalities encouraged several research groups to come up with identification approaches to facilitate the fast diagnosis of diseases based on image analysis techniques ${ }^{[3-10]}$. However, conventional image analysis techniques either use manual tracing approach or semi-automatic approaches that differ in the way the radiologist will intervene ${ }^{[5-10]}$. The requirement of automated image analysis methods combined with the development in computer industry motivated the researchers to propose new sophisticated image analysis techniques to challenge the automation problem ${ }^{[11]}$.

A number of groups have developed techniques for computer assisted segmentation of pulmonary CT images ${ }^{[3,5-10,12]}$. Hoffman et al. manually traced boundaries were used to estimate regional gas and tissue volumes in the lungs of normal subjects ${ }^{[8]}$. But manual methods are laborious and subject to both inter-observer and intra-observer variations. On two-dimensional (2D) transverse slices of a pulmonary CT dataset, the natural contrast between the low-density lungs and the surrounding high-density chest wall can be used to guide image segmentation. 2D edge tracking was used to find the boundaries of the left and right lungs. Others have used 3D region growing with manually specified seed points ${ }^{[5,8-10]}$. In many semi-automatic approaches, some manual interaction is required to select threshold values or edit the resulting segmentation ${ }^{[5-10]}$. In Kalender et al., anterior and posterior junction lines are provided manually to separate the right and left lungs in the case where the edge contrast is reduced by the volume averaging ${ }^{[6]}$. Brown et al. ${ }^{[12]}$ provided a knowledge-based, automatic method to segment chest CT images. In their method, anatomic knowledge stored in a semantic network is used to guide low-level image processing routines. Rather than requiring manual intervention to define the anterior junction lines as in Kalender et al. ${ }^{[6]}$, Brown et al. used dynamic programming to search for the junction lines automatically.

Shiying et al. ${ }^{[11]}$ developed a fully automatic method for identifying the lungs in CT images in 2001. The method has three main steps. First, the lung region is extracted from the CT images by gray level thresholding. The left and right lungs are then separated by detecting the anterior and posterior junction lines between the right and left lungs. Finally, smoothing the irregular boundary along the mediastinum is used to obtain more consistent results across time and leave lung structures with the lung. There are several distinctions between this method and previous work. First, instead of using a fixed threshold value, it used an optimal thresholding method ${ }^{[13]}$ to automatically choose a threshold value that reflects the grayscale characteristics of a specific dataset. Second, an efficient method was used to find an anterior and posterior junction lines between the right and left lungs. Finally, smoothing step was used to obtain more consistent results across time and to leave lung structures with the lungs.

Later in 2003, Aykac et al. ${ }^{[14]}$ designed an automatic algorithm for airway tree segmentation and analysis in CT images. Grayscale reconstruction is utilized to identify candidate areas on 2D slices. After this initial segmentation, bounded space dilation is applied to label connected airways and reconstruct the 3D airway tree after the tree structure has been established, tree branch segments and tree branch points can be identified. The method consists of the same steps discussed in Shiying et al. ${ }^{[11]}$.

There are some limitations on the methods presented ${ }^{[3,5-14]}$ to be directly applicable for detection. These papers are limited in their scope of work whereas they are focusing on presenting a way for airway tree segmentation and segmentation of the lung tissue and separate it from other surrounding regions. They don't consider any application for the lung and airway segmentations in diagnosis or detection of the abnormalities in the lung tissues. These papers can be utilized as starting point for the proposed work.

Armato et al. developed an automated method for the detection of lung nodules in helical CT images ${ }^{[15]}$. This technique incorporates 2D and 3D analyses to exploit the volumetric image data acquired during a CT examination. They tried to use 
the lung segmentation operation to capture lung nodules, however, detecting small nodules is not enough to lead for a real diagnosis, the radiologist needs also the characteristics of each detected nodule which is not presented in this work.

Satoh et al. developed a CAD system for lung cancer to detect nodule candidates at an early stage from the present and the early helical CT screening of the thorax ${ }^{[16]}$. In their work, they concentrated on coming up with a system that is able to detect small nodules, however, their work didn't consider classification of detected nodules or presenting any features to enable its diagnosis.

Performance evaluation of an automated classifier was conducted by Armato et al. to differentiate malignant and benign lung nodules in low-dose helical CT scans acquired as part of a lung cancer screening program ${ }^{[18]}$. In this work, Armato et al. proposed a fully automated computerized method for the detection of lung nodules in CT scans was presented to identify lung cancers that may be missed during visual interpretation ${ }^{[18]}$. Lung nodule identification proceeds in three stages: 2D processing followed by 3D analysis and application of an automated classifier. Awi et al. proposed and implemented a method of nodule detection for CT images ${ }^{[19]}$.

A CAD program that can read a CT data set and automatically detect pulmonary nodules on the images is further investigated ${ }^{[20]}$. The system should better performance in small nodules. The developed CAD systems usually focus on detecting small nodules that may be missed during visual interpretation ${ }^{[17-20]}$. Those works provide a CAD system that didn't consider the different features that will help the radiologist to speed up his/her diagnosis and reduce his/her workload. Radiologists don't only need 2D and 3D measurements of detected nodule but also need a short list of data that explains its internal morphology, size, location, and border shape.

It is noteworthy that, in this research, the current proposed CAD system will focus on developing a computerized system that has the advantage of detecting lung nodules and display its statistical features besides detecting the abnormal regions in the lung tissue and find its diagnostic measurements in order to help the radiologist to come up with an accurate diagnosis. It is expected that the proposed technique will increase diagnostic confidence of lung cancer.

\section{Methodology}

\subsection{Database construction and diagnosis validation}

The validation database of this study is consisted of 45 thoracic patients. These patients are scanned using CT scan during the interval of April 26th, 2005 to May 15th, 2011 at King Hussein Cancer Center. Forty of these patients were men, and five patients were woman. A bronchoscopy with histopathological study was conducted, which revealed a cancer was present. The age range of the patients at the time of the examinations was in the range of 52 years -74 years (mean age, 62 years). Each patient was named with a number to maintain their privacy. The data is divided into two sets: the first set is the training data set. It consists of $80 \%$ of the data (36 patients). It is used to train the classifier for proper modeling. The second set is the validation data set. It consists of $20 \%$ (nine patients). The validation data is used to show the performance of the proposed classifier.

The CT examinations were performed with a CT scanner (CT-S5VA47A; SIEMENS Medical Systems, Germany). Each reconstructed CT section had an image matrix size of $512 \times 512$ pixels. Forty Five scan sets in the database comprise a total of 2,921 images, with the number of images per set ranging from 45-83 images, with a mean of 65 images per set.

The visual diagnosis of the CT images was performed twice. The first time was conducted by the King Hussein Cancer Center team at the time of scanning and the second diagnosis was later performed at Jordan Hospital in order to maximize the efficiency and accuracy of the built system. 


\subsection{I mplementation of the automated system to detect and classify primary lung cancer}

The built CAD system works as a second reader for the CT scans images; the system converts the CT DICOM images from 12 into 8 bits stored Bitmap images. Threshold labeling is performed to segment the lung, the thresholding approach is based on Otsu thresholding algorithm that aims at minimizing the variance within the group. Now the binary image is subjected to Run-Length encoding labeling algorithm to label each detected region inside the lung tissue. Finally a set of statistical measurements and histogram features are applied to the detected regions to find its length in the anterior-posterior direction, width in the transverse direction, radius, area, spatial histogram, density histogram and the morphological feature of its boundary. By comparing and noticing the region characteristics, the radiologist can identify, detect, and classify the nature of the detected region whether it's a normal region or not. Figure 1 shows the block diagram of the automatic detection and classification algorithm.

Figure 1. Flow diagram illustrates the overall scheme for automated system to detect and classify primary lung cancer on CT images

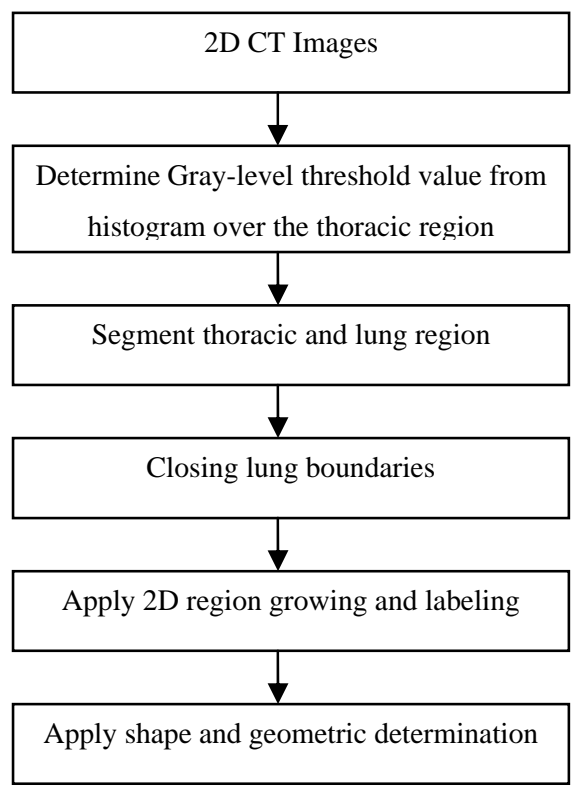

\subsubsection{Threshold labeling}

The simplest way to recognize the object regions is to perform a threshold labeling operation in which each pixel that has a high enough value is given the value binary 1 . The value binary 1 designates that the pixel has some possibility of being part of an object of interest. Each pixel that doesn't have a high enough value is given the value binary 0 . This designates that it has some possibility of being part of an object of interest. The thresholding technique used in this work is based on finding a threshold that minimizes the within group variance (Otsu threshold) ${ }^{[21]}$. If the histogram is bimodal, the histogram thresholding problem is to determine a best threshold $t$ separating the two modes of the histogram from each other. Each threshold $t$ determines a variance for the group of values that are less than or equal to $t$ and a variance for the group of values greater than $t$. The definition for best threshold suggested by Otsu is that threshold which the weighted sum of group variances is minimized. The weighs are the probabilities of the respective groups. The best threshold $t$ can then be determined by a simple sequential search through all possible values of $t$ to locate the threshold $t$ that minimizes $\sigma_{w}^{2}(t)$. In many situations this can be reduced to a search between the two modes. However, identification of the modes is really equivalent to the identification of separating values between the modes.

The binary image that is produced by the Otsu threshold is then subjected to manually selection of the lung tissue in order to omit and neglect the regions that are surrounding the lung tissue and out of the region of interest. The selection is based on selecting a binary-1 pixel in the lung region and based on 4-adjacency relation; the system captures all binary-1 connected pixels to the pixel being selected and omits others. Figure 2 shows the binary image with the regions needed to be processed. 


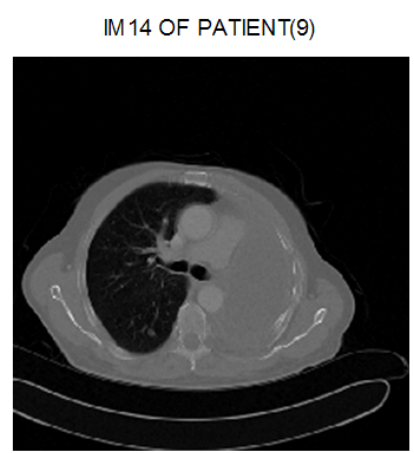

IM14 WITH LABELED REGIONS
IM14 AFTER THRESHOLDING

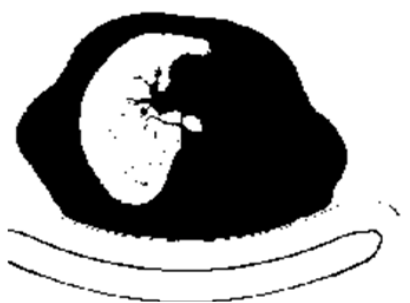

IM14 WITH FILLED LABELED REGIONS
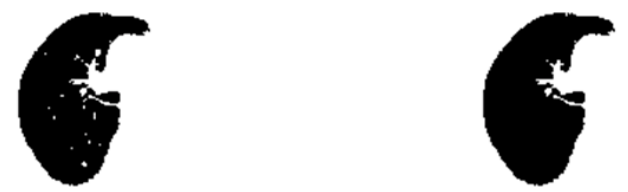

Figure 2. Efficiency of the thresholding and labeling operation, Patient_9 (IM14)

\subsubsection{Connected component labeling}

The connected components labeling operation performs the unit change from pixel to region. All pixels that have value binary- 1 and are connected to each other by a path of pixels all with value binary- 1 are given the same identifying label. The label is the identifier for a potential object region. Connected component labeling is a grouping operation ${ }^{\text {[21] }}$. The labeling operation used in this algorithm is based on Run-length encoding of the binary image ${ }^{[22]}$. This algorithm starts with Run-length encode of the input image then scan these runs and assigning preliminary labels and recording label equivalences in a local equivalence table before resolving the equivalence classes and finally re-label the runs based on the resolved equivalence classes.

\subsubsection{Feature extraction}

The analysis phase consists of computing global properties for each region produced by connected components labeling algorithm. The properties of each region that are considered by the radiologist while reading the CT image in order to detect primary lung cancer are the area of that region, its width and length, radius, morphological feature of its boundary and finally its spatial histogram. These measurements are made available for each region so that radiologist will be easily make his/her decision regarding the considered lesion.

All the properties that are needed by the radiologist to classify the founded regions from a CT image are computed automatically by the proposed system. This system takes the advantage of displaying the shape of the region boundary that is hard on the radiologist to see it clearly using the CT film. Also this system allows the radiologist to study the homogeneity of that region as well as displaying a numeric numbers of the region length, width, radius and area that will help the radiologist to come up with more accurate decision and diagnosis of the founded regions by minimizing the errors that result from manually measurements of the region properties and also reduce the errors resulted from inter and intra observer variation.

To test the performance of the proposed CAD system, 532 images that was obtained from the validation set is used. The images include 2,152 nodules. The specificity, accuracy, precision and recall of the system are calculated using the following formulas:

$$
\text { Specificity }=\mathrm{TN} /(\mathrm{TN}+\mathrm{FP})
$$




$$
\begin{gathered}
\text { Accuracy }=(\mathrm{TP}+\mathrm{TN}) /(\mathrm{TP}+\mathrm{FP}+\mathrm{TN}+\mathrm{FN}) \\
\text { Precision }=\mathrm{TP} /(\mathrm{TP}+\mathrm{FP}) \\
\text { Recall }=\mathrm{TP} /(\mathrm{TP}+\mathrm{FN})
\end{gathered}
$$

Where, TP: means true positives representing regions that are detected by the proposed system and classified by the two hospitals as positive; FP: means false positives representing regions that are detected by the proposed system and classified by the two hospitals as negative; FN: means false negatives representing regions that are missed by the proposed system and classified by the two hospitals as positive; TN: means true negatives representing regions missed by the proposed system and classified by the two hospitals as negative. The values for TP, FP, FN, and TN are determined by the experts of the two hospitals. When both hospitals agree on the result it is used in performance measures.

\section{Results}

To demonstrate the accuracy and effectiveness of the proposed algorithm a randomly selected CT image for a patient is used to show the flow of work of the algorithm. Figure 2 shows the ability of the thresholding and labeling algorithms in determining the actual areas without any loss of representation. To visually observe the accuracy of detection and localization, each labeled area is filled with zeros and projected on the original image. As it clearly appears in the figure, the run-length algorithm was able to detect and localize all possible areas without any losses. The proposed approach is though can be effective in localizing the diseased areas and enable the feature extraction algorithm to highlight their influence on the patient's medical record.

The internal morphology of the detected area is a significant indication that enables the radiologist to perform his/her diagnosis. Regions having heterogeneous areas are possible indications of the presence of necrosis, cavitations or calcification. To demonstrate the ability of the proposed system in showing such behavior of the detected tissue, the spatial histogram of the CAD system is used to study the homogeneity of each detected region. Figure 3 illustrates the different characteristics for the area indicated by a red star in the Figure. To demonstrate the capability of the system to detect small nodules as well as it is capability of detecting large nodules. Figure 4 shows the margins of the red star labeled area.

Table 1. Values for the performance parameters of the proposed system

\begin{tabular}{lll}
\hline Measured/Predicted & Negative & Positive \\
\hline Negative & $362(\mathrm{TN})$ & $13(\mathrm{FP})$ \\
Positive & $7(\mathrm{FN})$ & $368(\mathrm{TP})$ \\
\hline
\end{tabular}

Table 2. Performance indicators

\begin{tabular}{ll}
\hline Performance Indicator & Percentage Value (\%) \\
\hline Specificity & 96.53 \\
Accuracy & 97.33 \\
Recall & 98.13 \\
Precision & 96.59 \\
\hline
\end{tabular}

To validate the results obtained from the automated system and the radiologists' results, suspicious lesions for the 45 patients are screened by the radiologists of both King Hussein Center and Jordan Hospital. The results obtained for the $80 \%$ are used as training set, while the other $20 \%$ (nine patients) are used as validation set. Table 1 shows the performance parameters for the proposed system. Table 2 shows the performance indicators of the proposed systems. 
The same images are processed by the algorithms. The results show that the algorithm was able to detect and classify all the tested lesions with a classification accuracy of $100 \%$. The mean error in detecting lengths was $0.4 \pm 0.1 \mathrm{~mm}$, and the error in the area was $1+0.4 \mathrm{~mm}^{2}$.
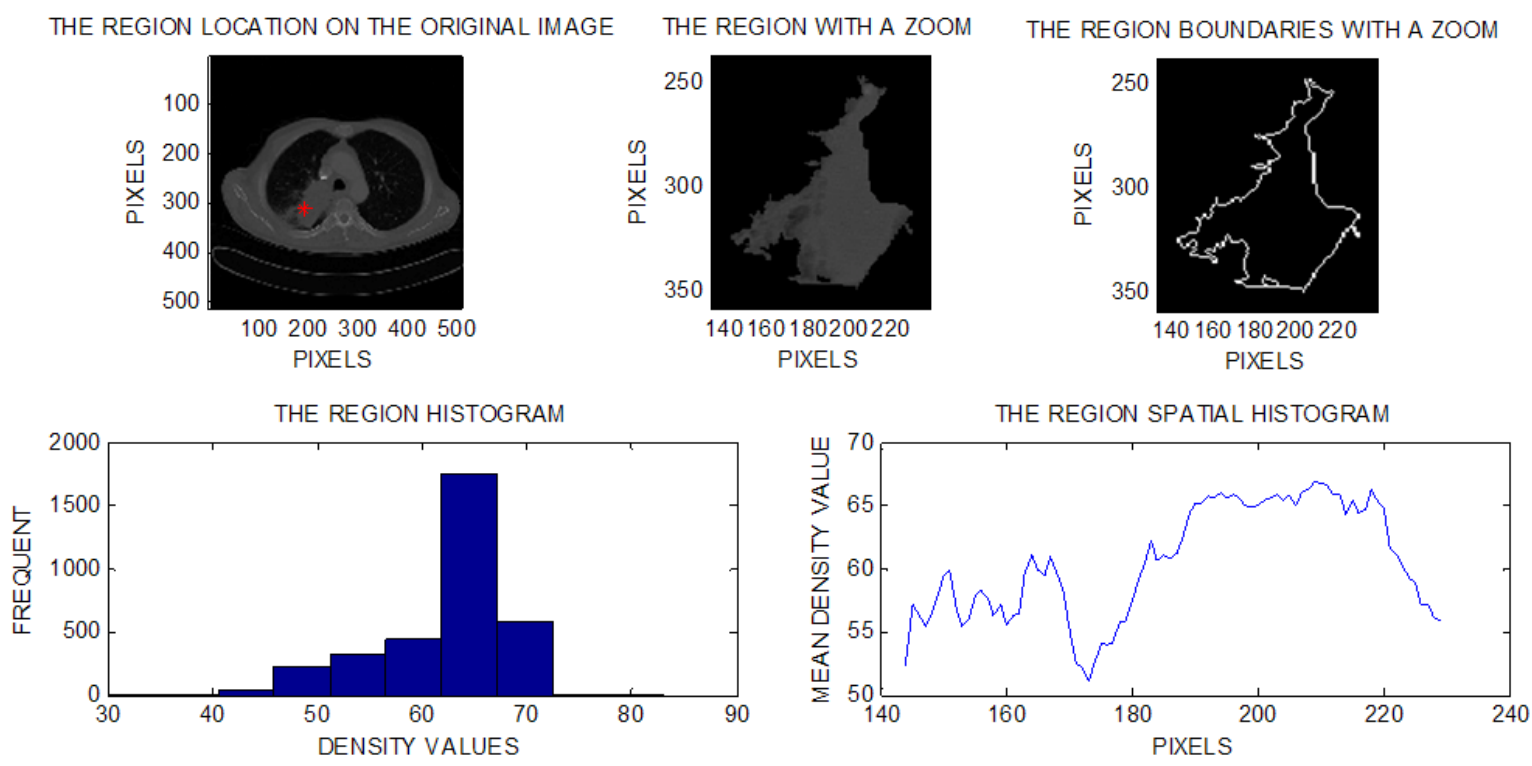

Figure 3. Characteristics of the red star indicated the upper half side of the figure
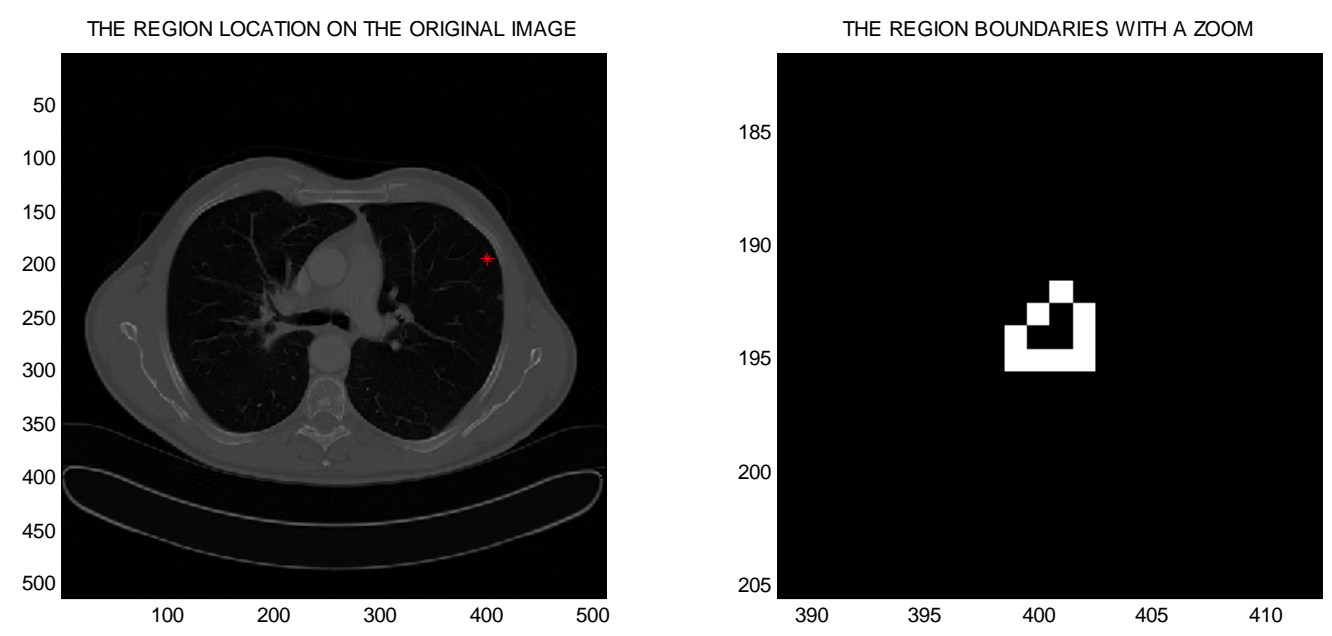

Figure 4. Margins of a detected nodule (1) Patient_3 (IM30)

\section{Discussion}

To discuss and highlight the strengths and weaknesses of this work, a comparative study with similar works available in the literature is performed. To compare the proposed system with the work proposed by Shiying et al. ${ }^{[11]}$, the system discussed in this study is able to find out the threshold value of each selected CT image automatically based on Otsu algorithm which shows an effective detection of the lung corresponding pixels. Before applying the 3D connected components labeling, a manually closing of the lung boundary is applied in order to close the regions located at the border of lung and extend into the mediastinum region, and it is one of the limitations that may be encountered while running the proposed system. Manually closing of the region may include pixels from the mediastinum region, which may lead to unbiased reduction of the accuracy of the estimated measurements of the system. However, manually closing when 
applied carefully to the lung boundaries it will minimize the errors that may be produced. Moreover, the proposed system deals with the whole image without the need to separate the lung into left or right regions as discussed in Shiying et al. It is noteworthy that the segmentation operation discussed in this work is not a fully automated segmentation as it includes some manual selection and closing operations. In comparison with the results obtained by Shiying and his team ${ }^{[11]}$ the segmentation operation discussed in this study is acceptable and show a high accuracy in detecting lung pixels during the thresholding operation. One hundred percent efficiency in labeling the regions inside the lung after carefully closing of the boundary regions is obtained.

Armato et al. presented a method for an automated lung nodule detection ${ }^{[18]}$. Ten percent of the 50 nodules in the database were not included in the initial set of nodule candidates due to improper extraction by the multiple-gray-level thresholding technique. However, in the proposed systems all the nodules are included with a high accuracy, precision, specificity, and recall percentages as it is clear in Table 2 .

Kazuo and his team studied the effect of Computer Aided Diagnosis on radiologist's detection performance ${ }^{[19]}$. The CAD system identified 62 and missed 16 nodules, yielding a true-positive rate of $80 \%$ in the total of 82 cases (total of 78 pulmonary nodules).

In Bae et al. ${ }^{[20]}$, the overall sensitivity for nodule detection method was $95.1 \%$. On the other hand, the specificity of the proposed algorithm is $96.53 \%$.

The classification results showed in other surveyed research are limited to the provided data that supported their detection ${ }^{[18-20]}$. Cancer areas are not easily configured due to its various shapes, locations, morphologies, pathological representation, and borders. Therefore, robustness and accuracy of the presented classification schemes cannot be guaranteed. Moreover, detected cancer nodules are not limited by size and/or location, many characteristics other than the location and the size of the nodule such as nodule margins and internal morphology are two other vital characteristics needed by the radiologist to finalize his/her diagnosis. The proposed CAD system in this work is able to provide the radiologist with many helpful diagnostic features that empower his/her conclusion about the detected area. Furthermore, the system displays the region size, margins, internal morphology as well as its location. Not only small nodules are detected but also masses and lesions are also characterized by the system.

The system accuracy is validated by conformance to the radiologist perception and its ability to get along with radiologist's readings. The detected nodules showed 98.13\% detection rate when compared with those results obtained by the radiologist. Double reading may increase the sensitivity, and CAD system is expected to aid radiologists in the detection of small nodules as well as displaying its features. The final diagnosis will be made by the radiologist, while CAD systems may serve to flag probable nodules when the images are reviewed, so that priorities can be set for interpretation of images. With rapid advances in computers and CT image acquisition technology, CAD techniques will achieve corresponding improvements in diagnostic accuracy and move closer to the clinical area.

The proposed CAD system is expected to provide an assistance tool to the radiologist helping him/her to identify very small defected area. It will help the radiologist to easily decide if the detected area is a cancer affected one or not. Moreover, it will be utilized to monitor the rate of change in which the tumor will grow where the system can detect small changes in size and displays the size of the detected lesion more accurately regardless of the direction of change that may be very difficult to be localized by manual selection or visual inspection. In addition, unguided rate of change may occur that cannot be manually measured. For successive CT scans for a patient during a period of time, the CAD system can measure the size of the detected nodules in each scan. For future scans the radiologist is able to compare the measured values with previous measures and decide the efficacy of any change. The system is able to measure changes in values less than $1 \mathrm{~mm}$. The system will be a valuable tool to assess the rate of improvement in the therapy process and would greatly help the medical doctor to pick the right medication especially at early stages of detection. 
The system was able to detect very small nodules that will not be easily observed by the radiologist and so can be utilized as a tool to study the occurrence of a nodule and the progress of the disease as function of time. This contribution is the most significant part of this work, where it demonstrates the ability of the proposed system in performing these tasks.

\section{Conclusions}

In this study an automated intelligent system for nodule detection and classification is proposed. The CAD system is able to read the DICOM CT images and to apply some advanced image processing principles to facilitate the segmentation and detection of mass lesions as well as small-sized cancer areas, presumably when they are biologically early in their evolution and amenable to surgical cure. The system is also able to display the size of the detected cancer, to replace the manually process of measurements that is taken by the radiologist to measure the width (transverse) and the length (anterior-posterior) distances of the cancer area. Detection of cancer region margins is a big challenge for the radiologist during diagnosis; margin is one of the features used during diagnosis to represent the growth of the tumor. The system discussed in this study displays each detected area boundary to simplify the detection of region boundary that is subjected to the observer variations. Low contrast cancer areas that have advanced stages in the disease may have calcification, necrosis and cavitations; low contrast regions constitute a challenge and a source of error for radiologist. The proposed system is able to display a histogram that enables the radiologist to study the homogeneity of the cancer area.

The built CAD system will play a vital role in detecting small changes in the size of the detected nodules, giving the radiologist the ability to study and measure the growth factor of the tumor over a period of time. It will assist the efficacy of therapy and help the medical doctor to select the right medication if it is used at the early stages of the disease.

\section{References}

[1] Cancer Prevention Directorate, Ministry of Health, Amman-Jordan, 2005-2011.

[2] Directorate of Information, Studies and Research, Ministry of Health, Amman-Jordan, 2005-2011.

[3] D. M. Denison, M. D. L. Morgan, A. B. Millar. Estimation of regional gas and tissue volumes of the lung in supine man using computed tomography. Thorax. 1986; 41: 620-628. PMid: 3787544. http://dx.doi.org/10.1136/thx.41.8.620

[4] M. Sonka, W. Park, E. A. Hoffman. Rule- based detection of intrathoracic airway trees. Comput. Med. Imag. Graph. 1996; 15: 314-326.

[5] L. W. Hedlund, R. F. Anderson, P. L. Goulding, J. W. Beck, E. L. Effmann, C. E. Putman. Two methods for isolating the lung area of a CT scan for density information. Radiology. 1982; 144: 353-357. PMid: 7089289.

[6] W. A. Kalender, H. Fichte, W. Bautz, M. Skalej. Semiautomatic evaluation procedures for quantitative CT of the lung. J. Comput. Assist. Tomogr. 1991; 15(2): 248-255. PMid: 2002103. http://dx.doi.org/10.1097/00004728-199103000-00011

[7] J. M. keller, F. M. Edwards, R. Rundle. Automatic outlining of regions on CT scans. J. Comput. Assist. Tomogr. 1981; 5(2): 240-245. PMid: 7217448. http://dx.doi.org/10.1097/00004728-198104000-00015

[8] E. A. Hoffman, E. L. Ritman. Effect of body orientation on regional lung expansion in dog and sloth. J. Appl. Physiol. 1985; 59(2): 481-491.

[9] E. A. Hoffman, L. J. Sinak, R. A. Robb, E. L. Ritman. Noninvasive quantitative imaging of shape and volume of lungs. J. Appl. Physiol (Resp., Environ., Exercise physiol.). 1983; 54(5): 1414-1421.

[10] E. A. Hoffman. Effect of body orientation or regional lung expansion: A computed tomographic approach. J. Appl. Physiol. 1985; 59(2): 468-480. PMid: 4030599.

[11] Shiying Hu, E. A. Hoffman, J. M. Reinhardt. Automatic Lung Segmentation for Accurate Quantification of Volumetric X-Ray CT Images. IEEE Trans. Med. Imag. 2001 Jun; 20: 490-498. PMid: 11437109. http://dx.doi.org/10.1109/42.929615

[12] M. S. Brown, M. F. McNitt-Gray, N. J. Mankovich, J. G. Goldin, J. Hiller, L. S. Wilson, et al. Method for segmenting chest CT image data using an anatomical model: Preliminary results. IEEE Trans. Med. Imag. 1997 Dec; 16: 828-839. PMid: 9533583. http://dx.doi.org/10.1109/42.650879

[13] M. Sonka, V. Hlavac, R. Boyle. Image processing, Analysis and Machine Vision. Pacific Grove, CA: PWS. 1999. 
[14] D. Aykac, E. A. Hoffman, G. McLennan, J. M. Reinhardt. Segmentation and Analysis of the Human Airway Tree From Three-Dimensional X-Ray CT Images. IEEE Trans. Med. Imag. 2003 Aug; 22: 940-950. PMid: 12906248. http://dx.doi.org/10.1109/TMI.2003.815905

[15] Armato SG III, Giger ML, Blackburn JT, Doi K, MacMahon H. Three-dimensional approach to lung nodule detection in helical CT. Proc SPIE. 1999; 3661: 553-559. http://dx.doi.org/10.1117/12.348611

[16] Satoh H, Ukai Y, Niki N, et al. Computer aided diagnosis system for lung cancer based on retrospective helical CT image. Proc SPIE. 1999; 3661: 1324-1335. http://dx.doi.org/10.1117/12.348530

[17] Armato SG III, Giger ML, MacMahon H. Automated detection of lung nodules in CT scans: preliminary results. Med Phys. 2001; 28: 1552-1561. PMid: 11548926. http://dx.doi.org/10.1118/1.1387272

[18] Armato SG III, Li Feng, Giger ML, MacMahon H, Sone S, Doi K. Lung Cancer: Performance of Automated Lung Nodule Detection Applied to Cancers Missed in a CT Screening Program. Radiology. 2002; 225: 685-692. PMid: 12461246. http://dx.doi.org/10.1148/radiol.2253011376

[19] Awai K, Murao K, Ozawa A, Komi M, Hayakawa H, Hori S, et al. Pulmonary Nodules at Chest CT: Effect of Computer-aided Diagnosis on Radiologists’ Detection Performance. Radiology. 2004; 230: 347-352. PMid: 14752180. http://dx.doi.org/10.1148/radiol.2302030049

[20] Bae K T, Kim Jin-Sung, Na Yong-Hum, Kim K G, Kim Jin-Hwan. Pulmonary Nodules: Automated Detection on CT Images with Morphologic Matching Algorithm- Preliminary Results. Radiology. 2005; 236: 286-294. PMid: 15955862. http://dx.doi.org/10.1148/radiol.2361041286

[21] Haralick, R. M., Shapiro, L. G. Computer and Robot Vision, Addison Wesley. Volume 1, USA, 1992.

[22] Gonzalez, R. C., Woods R. E., Eddins S. L. Digital Image Processing Using MATLAB, Pearson Prentice Hall, USA, 2004. 